\title{
Engineering synthetic optogenetic networks for biomedical applications
}

\author{
Meiyan Wang ${ }^{1}$, Yuanhuan $\mathrm{Yu}^{1}$, Jiawei Shao ${ }^{1}$, Boon Chin Heng ${ }^{2}$ and Haifeng $\mathrm{Ye}^{1, *}$ \\ 1 Shanghai Key Laboratory of Regulatory Biology, Institute of Biomedical Sciences and School of Life Sciences, East China \\ Normal University, Shanghai 200241, China \\ 2 Department of Endodontology, Faculty of Dentistry, The University of Hong Kong, Hong Kong SAR 999077, China \\ * Correspondence: hfye@bio.ecnu.edu.cn
}

Received December 7, 2016; Revised January 24, 2017; Accepted January 25, 2017

\begin{abstract}
Background: Recently, optogenetics based on genetically encoded photosensitive proteins has emerged as an innovative technology platform to revolutionize manipulation of cellular behavior through light stimulation. It has enabled user defined control of various cellular behaviors with spatiotemporal precision and minimal invasiveness, creating unprecedented opportunities for biomedical applications.

Results: This article reviews current advances in optogenetic networks designed for the treatment of human diseases. We highlight the advantages of these optogenetic networks, as well as emerging questions and future perspectives. Conclusions: Various optogenetic systems have been engineered to control biological processes at all levels using light and applied for numerous diseases, such as metabolic disorders, cancer, and immune diseases. Continued development of optogenetic modules will be necessary to precisely control of gene expression magnitude towards clinical medical practice in the context of real-world problems.
\end{abstract}

Keywords: synthetic biology; mammalian designer cells; optogenetics; synthetic gene circuits; gene- and cell-based therapy

\section{INTRODUCTION}

Over the past decade, synthetic biology has emerged as an interdisciplinary field that addresses crucial challenges in human health and disease with an engineering approach $[1,2]$. Synthetic biology provides the platform and tools to design artificial regulators to rationally and precisely control the biological activities of cells [3]. To date, many complex synthetic devices have been designed and constructed such as toggle switches [4], sensor-effector device [5], oscillators [6,7], pattern detectors [8], and intercellular communication systems [8,9]. Most of the devices were responsive to small molecule inducers and had inherent limitations in regard to precise spatiotemporal control of gene expression. Recently, optogenetics has emerged as an innovative technology platform in the rapidly progressing field of synthetic biology to achieve highly precise spatiotemporal control and manipulation of cellular behavior through light stimulation, both in vitro and in vivo [10]. In addition, the target gene expressions can also be quantitatively regulated by controlling the different light irradiances $[11,12]$. Some fine-tuned circuits based on bidirectional promoter were developed to quantitatively regulate multiple gene expression by modulating light irradiance [12].

The broad definition of optogenetics refers to combining optical and genetic principles together to control various cellular functions such as stimulation/inhibition of metabolic pathways [13,14], gene activation [15], intracellular signaling [16], and cellular migration [17] upon stimulation with a specific wavelength of light. Genetically encoded light-responsive systems exhibit various distinctive features, which make them stand out from other synthetic gene networks, such as fast activation and inactivation kinetics, low technical requirements for equipment and reagents besides illumination, and lack of side effects typically associated with drug administration. This technology platform has recently attracted much attention due to the unmatched properties of light as a signaling entity, which enables highly precise 
spatio-temporal and quantitative control of cellular activities and gene expressions.

Since the debut of optogenetics about a decade ago, the field has developed rapidly. The history of optogenetic networks [18-22] is summarized in Figure 1. The first described microbial opsin, bacteriorhodopsin, dates back to the 1970s [23,24]. Over the subsequent years, a diverse variety of other light-sensitive proteins have been identified, such as halorhodopsin and channelrhodopsin. It was not until 2005 that the concept of fast optogenetic stimulation was realized with reliable control of millisecond-precision action potentials in mammalian neurons through light stimulation [21,25]. In 2010, the journal Nature declared optogenetics to be their "method of the year" [26], while the journal Science classified it as one of the major breakthroughs of the last decade [27].

Recently, optogenetics has turned early ideas into a powerful paradigm for cell biology $[28,29]$. In the most recent studies, several light-responsive gene expression systems designed with the engineering-driven approaches of modularization, rationalization and modeling have been created to monitor and control mammalian cellular activities with the unmatched precision of light stimulation [30-34]. These provide a trajectory for future development in the field. This review therefore aims to highlight the latest state of-the-art optogenetics-associated light-responsive synthetic networks for controlling gene expression in some important biomedical applications.

\section{OPTOGENETIC NETWORKS FOR THE TREATMENT OF DIABETES MELLITUS}

Type 2 diabetes is a chronic metabolic disease characterized by hyperglycemia and results from the combination of resistance to insulin, inadequate insulin secretion, and inappropriate glucagon secretion [35,36]. Diabetes has afflicted increasing numbers of people worldwide. Most people with type 2 diabetes will eventually need to inject insulin and take other medications, as well as strictly control their diet [37].

Recently, a number of promising optogenetic networks have been developed for the treatment of diabetes (Figure 2). In a pioneering study by Ye et al. [38], a blue-light responsive gene switch was created in human cells to activate transgene expression in an animal model (Figure 2A). In this design, a novel synthetic light-controlled transcription device could be used to treat type II diabetes. Under stimulation with blue light $(\sim 480 \mathrm{~nm})$, melanopsin activates the nuclear factor of activated T cells (NFAT) through elevated calcium levels, which induces glucagon-

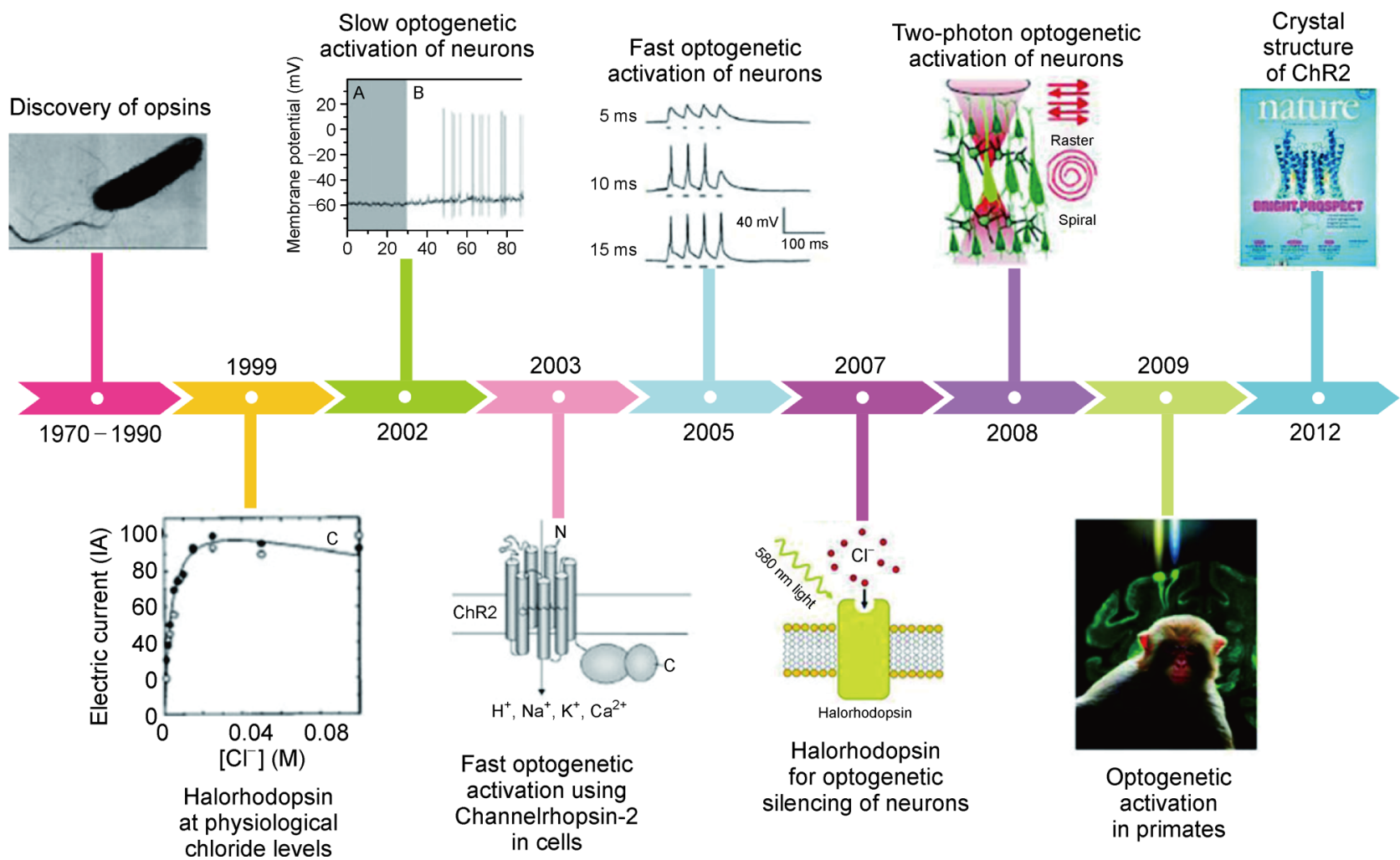

Figure 1. A brief summary of the history of optogenetics. 1999 - Reprinted with permission from Ref. [8]; 2002- Reprinted with permission from Ref. [9]; 2003 - Reprinted with permission from Ref. [10]; 2005 - Reprinted with permission from Ref. [11]; 2012 Reprinted with permission from Ref. [12]. 


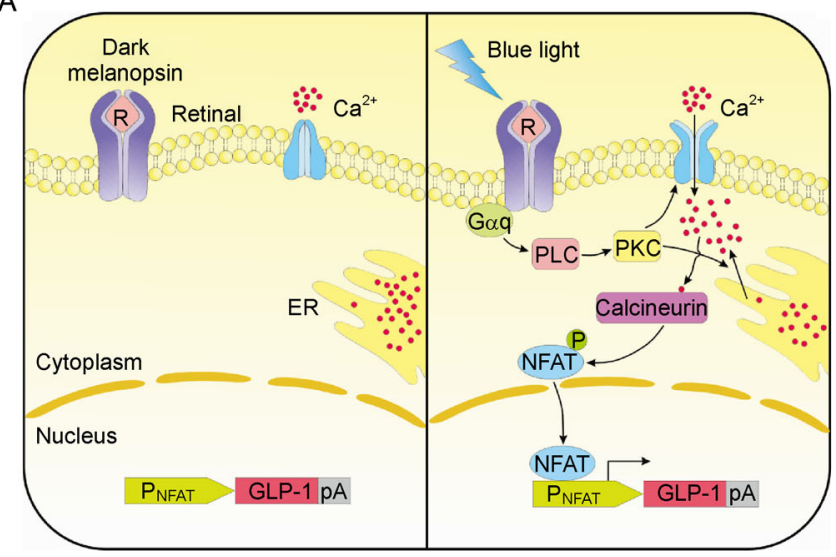

$\mathrm{B}$

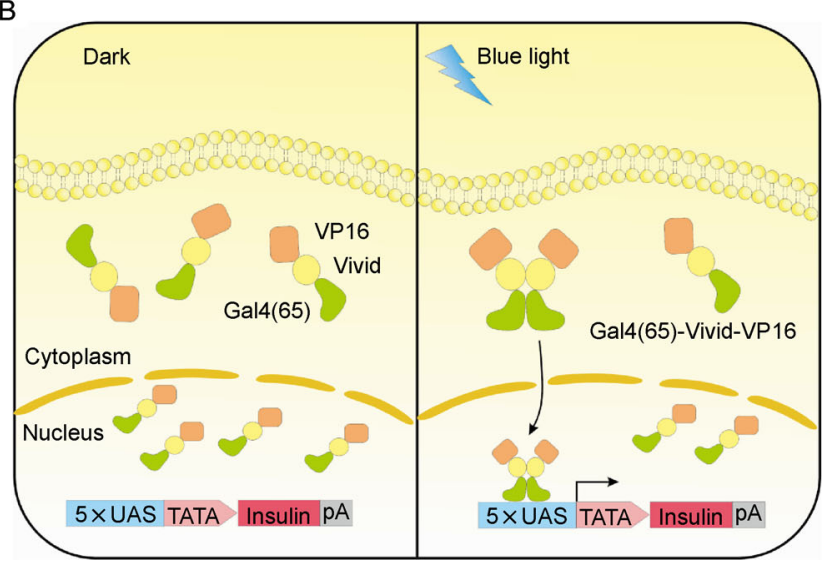

Figure 2. Synthetic optogenetic networks for diabetes therapy. (A) Synthetic optogenetic network for modulating glucose homeostasis in diabetic mice. In the dark, melanopsin do not trigger the phosphodiesterase-dependent cascade. Under blue-light illumination, the 11-cis retinal $(\mathrm{R})$ chromophore is isomerized and changes the conformation of melanopsin, thus sequentially activating the Gaq-type $G$ protein (Gaq), phospholipase $C(P L C)$, and phosphokinase $C(P K C)$, which in turn triggers calcium influx by transient receptor potential channels (TRPCs). The increased intracellular calcium levels activate the calcium sensor protein calmodulin (CaM) and calcineurin (CaN) and induce glucagon-like peptide-1 (GLP-1) expression via an NFAT-responsive promoter $\left(P_{\text {NFAT }}\right)$. The transgenic HEK-293 cells containing this synthetic gene circuit were microencapsulated and subcutaneously implanted into diabetic mice, which lowered the blood-glucose levels after blue-light exposure. (B) A light-controlled transgene system based on light-oxygen-voltage (LOV) domain for the treatment of diabetes. In the dark, GAVP do not interact with UAS $_{\mathrm{G}}$ elements $\left(5 x \cup A S_{G}\right)$ and do not induce gene expression. After blue light activation, Vivid (VVD), one of the light-oxygen-voltage (LOV) domain, changes its structure to form a dimer and induces dimerization of the Gal4(65)-VVD fusion protein, which enhances binding to the $\mathrm{UAS}_{\mathrm{G}}$ sequence and activates transcription of insulin. The system was transferred into type I diabetic mice by a hydrodynamic procedure, and the blood glucose levels were significantly decreased under blue-light illumination.

like peptide 1 (GLP-1) expression through a NFAT driven promoter. This can attenuate glycemic excursions in type II diabetic patients. When engineered cells containing this synthetic light-responsive network were subcutaneously implanted into type II diabetic mice, control of bloodglucose homeostasis was achieved by blue light stimulation.

In another study, Wang et al. [39] designed a lightcontrolled transgene system as a triggering mechanism to control insulin expression for type I diabetes treatment (Figure 2B). In this system, the light-oxygen-voltage domain Vivid (VVD) was used to control gene expression by blue light. The light-switchable transgene system contained VVD, a monomeric variant of the Gal4-DNAbinding domain and the activation domain p65. Upon stimulation with pulses of blue light, VVD can change its structure to form a dimer, thus dimerizing the Gal4-DNA binding domain to bind to its cognate promoter and activating insulin transcription. The inactive state was however maintained in the dark. When the system was transferred into type I diabetic mice by a hydrodynamic procedure, the blood glucose levels were significantly decreased under blue-light illumination. This light responsive system provides another example of controlling biological processes through light-switchable gene expression.

\section{OPTOGENETIC NETWORKS FOR ONCOTHERAPY}

Targeting the immune system to combat tumors is a promising therapeutic strategy [40]. T-cells are removed from a patient and modified, and then reintroduced back into the patient with the ability to recognize and kill targeted cancer cells [41-43]. Recently, optogenetic systems have been developed to enable immune cells to specifically attack tumor cells (Figure 3).

In the study of Zhou et al. [44], a near-infrared (NIR)responsive optogenetic system (termed "Opto-CRAC") was designed as a tool for immunomodulation and immunotherapy, by remotely controlling $\mathrm{Ca}^{2+}$-responsive gene expression in specialized immune cells, such as $\mathrm{T}$ lymphocytes, macrophages and dendritic cells (Figure 3A). In this study, the NIR-responsive optogenetic platform based on lanthanide-doped upconversion nanoparticles (UCNP) and the engineered calcium channel (Opto-CRAC channels) can remotely control engineered cells buried deep within tissues by UCNP turning NIR light into visible light. The engineered CRAC channels consisted of ORAI1 and engineered stromal interaction molecule 1 (STIM1, ER-located $\mathrm{Ca}^{2+}$ sensor protein) fused with the light switch LOV2 (light, oxygen, voltage) domain. Upon illumination with blue light, C-terminal J $\alpha$ 
A

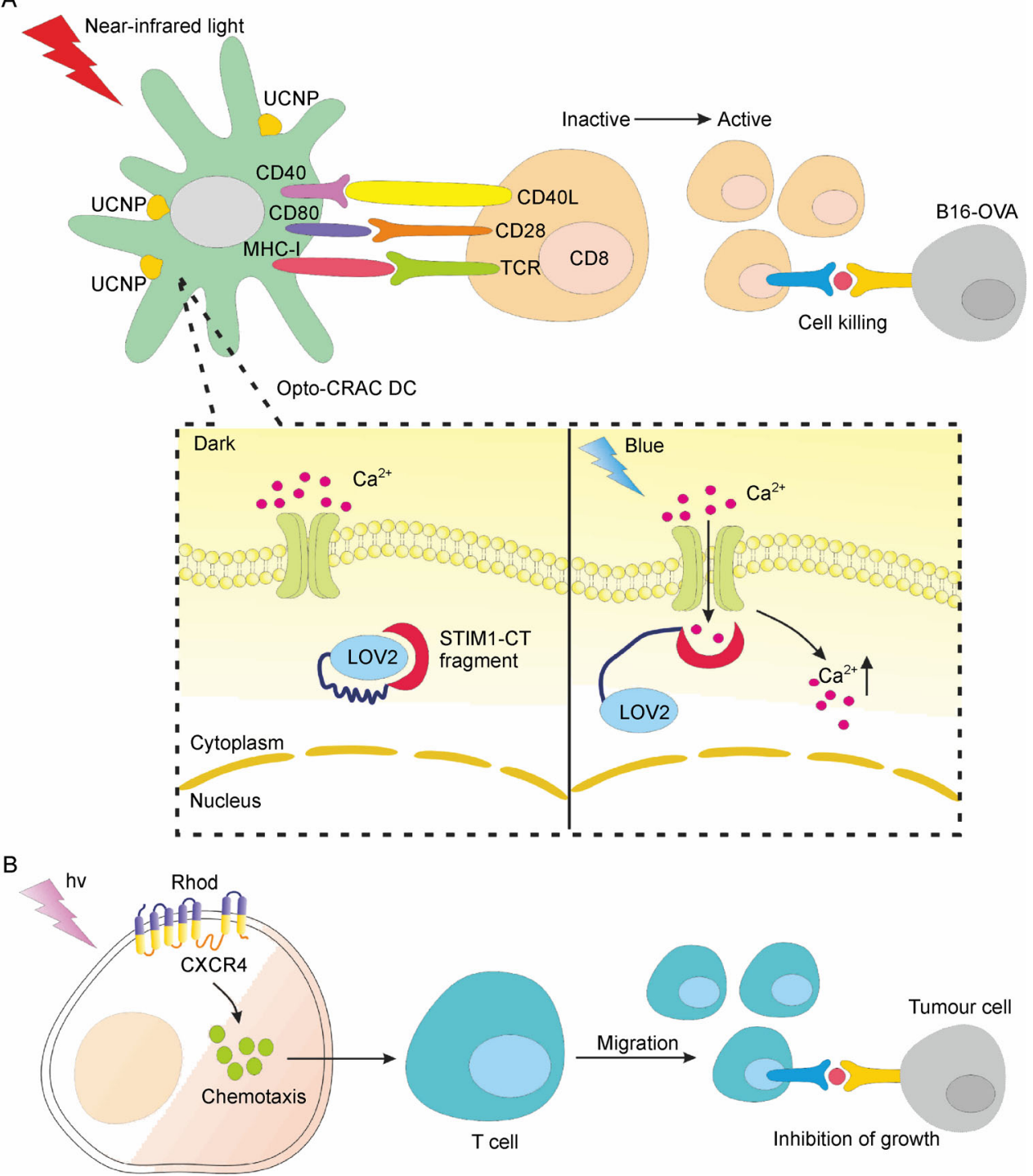

Figure 3. Synthetic gene circuits for cancer therapy. (A) A synthetic near infrared (NIR)-stimulable optogenetic platform (termed Opto-CRAC) designed for treatment of melanoma in a B16-OVA murine model. Photosensitive STIM1 can be engineered by fusing STIM1-CT fragments with the LOV2 (light, oxygen, voltage) domain (residues 404-546) of Avena sativa phototropin 1. In the dark, STIM1-CT fragments are kept quiescent by docking toward the LOV2 domain. Upon blue light illumination, the undocking and unwinding of the LOV2 C-terminal Ja helix exposes the STIM1-CT fragments, enabling their interaction with ORAI1 Ca ${ }^{2+}$ channels to induce calcium ion influx across the plasma membrane. When coupled to lanthanide-doped upconversion nanoparticles (UCNP), the optogenetic excitation window is converted to visible light from tissue-penetrable NIR light to enable wireless photo activation of $\mathrm{Ca}^{2+}$-dependent signaling and modulation of immune cells activities. NIR-stimulated calcium ion influx in Opto-CRAC DCs facilitate immature dendritic cell maturation and promote antigen presentation, thereby sensitizing $T$ lymphocytes towards tumor antigens to improve anti-tumor immune response to selectively kill tumor cells in the B16-OVA melanoma model. (B) Optical control of chemokine receptor used for cancer immunotherapy in B16/OVA tumor bearing mice. Under $505 \mathrm{~nm}$ light irradiation, a photoactivatable chemokine receptor CXCR4 (PA-CXCR4) based on rhodopsin and chemokine receptor is activated to recruit transferred T cells and make them migrate into the core of B16 tumors, promoting local effector functions and suppressing tumor growth.

helix of LOV2 was unwinded to enable mobility of STIM1-CT fragments, resulting in activation of ORAI1 $\mathrm{Ca}^{2+}$ channels and influx of calcium ions into the cells, which in turn activate calcium dependent gene expression to elicit a series of immune responses. Additionally, upon injection of the Opto-CRAC system into the mouse model 
of melanoma by utilizing ovalbumin as an antigen, the system can elicit anti-tumor immune responses to specifically kill tumor cells. This approach will pave the way for broader applications of optogenetic tools in synthetic biology that enables the remote and wireless regulation of gene networks.

Another study [45] also reported an optical chemokine receptor system based on photoactivatable-chemokine C-X-C motif receptor 4 (PA-CXCR4) that can induce highly specific chemokine signals and guide cell migration through light stimulation (Figure 3B). The genetic circuit was carefully designed to couple an extracellular optical signal to intracellular chemokine effector functions, which in turn can control chemotactic signals and modulate directional cell migration towards the source of light stimulation. This synthetic chemotaxis system was validated in a mouse model. PA-CXCR4 was shown to recruit transfused tumor-specific $\mathrm{CD} 8^{+} \mathrm{T}$ cells to a tumor site in vivo, resulting in significant reduction of tumor growth in mice after light stimulation. These findings thus suggest that photoactivatable chemokine receptors provide a unique platform to remotely control cell trafficking to enable accurate lymphocyte chemotaxis and thus gain access to a specific tissue site in response to localized stimulation with a specific light wavelength. The utilization of antitumor T-cell migration to localize chemokine signals for treatment of tumors was also confirmed by another study $[46,47]$.

\section{OPTOGENETIC NETWORKS FOR THE TREATMENT OF RETROGRADE AMNE- SIA}

Retrograde amnesia is a form of memory loss in patients with closed head injury, Korakoff's syndrome, and other memory disorders, in which remote memories are more easily accessible than events occurring just prior to the trauma. Retrograde amnesia usually follows damage to areas of the brain other than the hippocampus that deal largely with memory consolidation [48-50], because existing long-term memories are stored in the neurons and synapses of various different brain regions. Memory consolidation is the phenomenon characterized by newly formed memory transitions from a fragile state to a stable and persistent state.

Recently, several groups demonstrated that specific activation of hippocampal cells for encoding memory are both sufficient and necessary to drive future recall of a contextual fear memory and thus represent a component of a distributed memory engram [51-56]. Tonegawa et al. [57] designed an optogenetic stimulation system based on channelrhodopsin-2 (ChR2). In this study, dentate gyrus (DG) and CA3 engram cells were labeled with TREChR2-EYFP and AAV9-TRE-mCherry in amnesic mice induced by a protein synthesis inhibitor. Under light illumination, DG engram cells were induced to restore retrograde amnesia caused by disruption of reconsolidation of a contextual fear memory [58], and the physiological connectivity between DG and CA3 or basolateral amygdala (BLA) engram cells was activated. Together, these findings suggest that engram cells induced by contextual fear conditioning (CFC) training are crucial for retrieving memory recall.

\section{OPTOGENETIC NETWORKS FOR THE TREATMENT OF PENILE ERECTION}

Precise spatiotemporal control of physiological processes by optogenetic networks may provide a strategy for geneand cell based therapies. Optogenetics has become a key technology to modulate neuronal activities managing memory [52], learning [59], and olfactory processing [60]. Penile erection is the result of a complex neurovascular process involving various central and peripheral neural and/or humoral endocrine mechanisms $[61,62]$. It is widely accepted that nitric oxide plays an important role in penile erection [63-65]. Nitric oxide stimulates soluble guanylyl cyclase (sGC), subsequently inducing the second messenger 3', 5'-cyclic guanosine monophosphate (cGMP) to reduce intracellular calcium ion concentration by closure of voltage-gated calcium channels, which leads to relaxation of the smooth muscle of the corpus cavernosum and triggering penile erection [66-68].

Erectile dysfunction is a sexual dysfunction affecting more than 100 million people worldwide. Erectile dysfunction can occur due to both physiological and psychological reasons including diabetes, kidney disease, chronic alcoholism, multiple sclerosis, atherosclerosis, vascular disease, and neurologic disease [69-72]. cGMP specific phosphodiesterase inhibitors such as Viagra ${ }^{\circledR}$ have been used to treat erectile dysfunction by suppressing cGMP and extending erection time. However, these drugs often exert adverse side effects in some patients suffering from hypotension or severe hepatic dysfunction and heart disease, which thus limited their clinical applications. With the advent of synthetic biology, genetic devices can provide an alternative method to precisely control the physiological process of this particular disease, thus providing novel therapeutic strategies for erectile dysfunction.

Recently, Kim et al. [73] designed and validated a bluelight-responsive synthetic erectile optogenetic circuit in human cells (Figure 4). This erectile optogenetic stimulator (EROS) biosynthesizes guanylate cyclase acid upon blue-light illumination, leading to high levels of cGMP in mammalian cells and reduced intracellular calcium levels. When introducing EROS to the corpus 
cavernosum of male rats, the treated animals exhibited obvious erectile response and ejaculation upon exposure to blue light. Compared with other methods [74-78], the EROS device provides trigger-inducible erection on demand with less side effects, but needs to be evaluated in clinical trials for the treatment of erectile dysfunction.

\section{OPTOGENETIC NETWORKS FOR ORGA- NELLE TRANSPORT}

Peroxisomal disorders represent genetically heterogeneous metabolic diseases characterized by the absence of normal peroxisomes in the cells of the body. Peroxisomes are cellular organelles involved in important metabolic pathways, such as beta-oxidation of very-longchain fatty acids (VLCFA) and detoxification of hydrogen peroxide [79]. Impaired peroxisome transport causes compromised peroxisome function resulting in oxidative stress in many neurodegenerative diseases [80]. Recently, optogenetic networks have been developed to directly regulate protein trafficking, including nuclear-cytoplasmic trafficking using light (Figure 5) [81-84].

Spiltoir et al. [85] used blue-light-responsive LOV2 domain of Avena sativa phototropin1 (AsLOV2) to directly control peroxisomal import with light. They developed caged GFP-LOV-PTS1 constructs containing a GFP reporter, AsLOV2-J $\alpha$ motif and peroxisomal targeting signal (PTS1) protein bound to the Pex5 peroxisomal import receptor to localize peroxisome with light (Figure $5 \mathrm{~A})$. When proteins or peptides are attached to the $\mathrm{C}$-terminus of the $\mathrm{J} \alpha$-helix, they can be blocked and could not interact with effectors in the dark, but the block can be uncaged upon illumination and Ja-helix unfolding. This system provides a tool to elicit peroxisomal protein trafficking at specific time points or within defined locations in specific cells through optical activation and deactivation.

Another example is optical control of organelle transport and positioning (Figure 5B) [86]. The function of various organelles depends on their proper spatial arrangement. Despite the importance of local positioning, the precise correlation between position and function is not yet fully understood. In order to solve this problem, many studies have developed different strategies to manipulate organelle distributions. Recent technical advances, such as optogenetics, are beginning to provide newer and more accurate ways to manipulate organelle positioning. One strategy is to control the motility of kinesin 5 and kinesin 1 in vitro by photocontrol ATP hydrolysis through incorporating photochromic molecules into the ATPase domain of motor proteins $[87,88]$. In addition, another study reported that both the speed and directionality of myosin VI and kinesin 14 motors could be modulated by post exposure to blue light [89]. However, these methods have not been applied to reposition organelles. Recently, Bergeijk et al. [86] have established a light-responsive intracellular transport system by using light-sensitive heterodimerization to control the connection between selected organelles and specific motor proteins such as kinesin, dynein or myosin. Under light illumination, the synthetic systems containing blue light sensitive LOV2 (light- oxygen-voltage-sensing) domain with an engineered PDZ domain and cryptochrome 2 together with CIB1 (cryptochrome-interacting basic-helix-loop-helix 1) can induce the motility of peroxisomes, mitochondria, and recycling endosomes. Selected areas from peroxisomes or mitochondria could be depleted by localized laser illumination, whereas

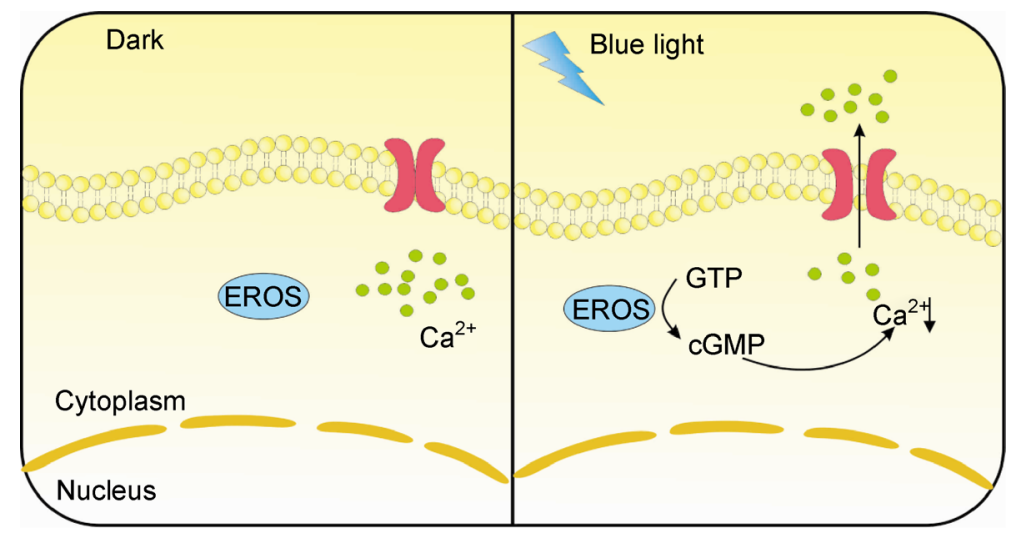

Figure 4. Synthetic erectile optogenetic stimulator for the treatment of male erectile dysfunction. A synthetic erectile optogenetic stimulator (EROS) containing a blue-light sensor domain BLUF (blue-light using FAD) activates an adjacent catalytic nucleotide cyclase domain. In the dark, EROS is inactivated. Under blue light illumination, the synthetic erectile optogenetic stimulator (EROS) enables conversion of GTP to cGMP, which activates protein kinase $\mathrm{C}$ to trigger $\mathrm{Ca}^{2+}$-outflux decrease by closure of voltage-gated calcium channels. This process ultimately results in the relaxation of the corpus cavernosum smooth muscle and subsequent penile erection. 
A

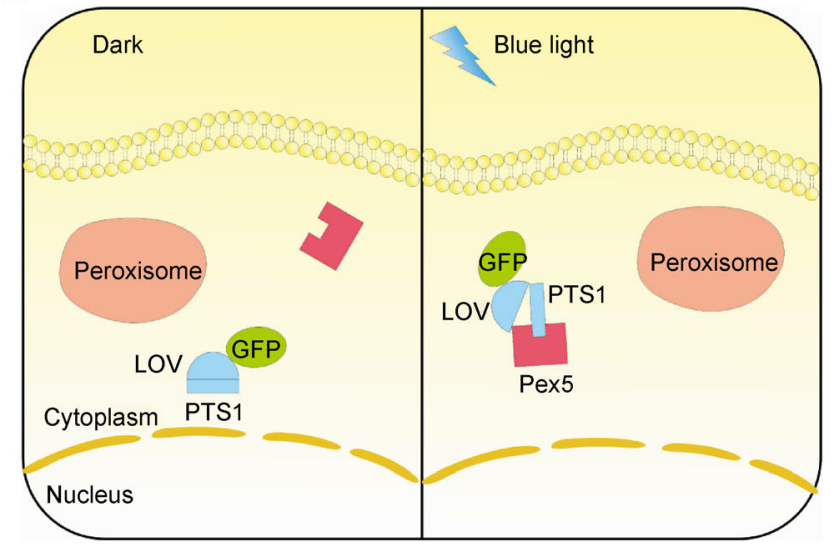

B

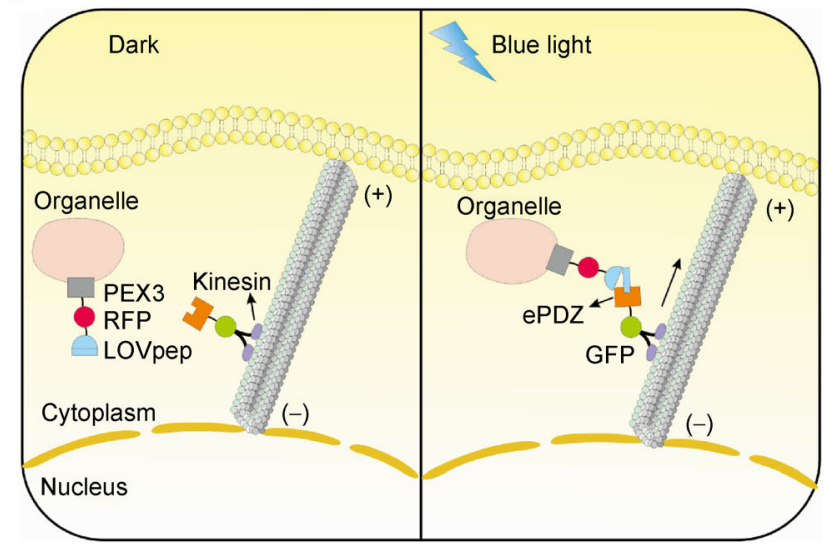

Figure 5. Optical control of organelle transport and positioning. (A) Optical control of peroxisomal trafficking based on LOV2 (light, oxygen, voltage) domain of Avena sativa phototropin1 (AsLOV2). LOV-PTS1 construct contains C-terminus of the Ja-helix that can be released from the LOV core in the presence of light and uncages the PTS1 sequence. When Pex5 binds to PTS1, the protein is translocated to the peroxisome. (B) Local and reversible activation of organelle transport with light. PEX-LOV fusion protein consists of a peroxisomal targeting signal of PEX3 and a photosensitive LOV domain. In the dark, the photosensitive protein is in the free state and do not control organelle positioning. Upon exposure to blue light, PEX-LOV cages a small peptide that binds the engineered PDZ domain ePDZb1 fused to the plus-end-directed kinesin-3 KIF1A, leading to relocation of peroxisomes from the cell centre to the periphery.

recycling endosomes may be induced to enter into specific dendritic spines, targeted away or moved towards axonal growth cones. A subsequent study extended this optogenetic tool to lysosomes [90]. Different optogenetic systems can be used to manipulate organelle positioning with precise spatiotemporal control.

\section{OPTOGENETIC GENE TARGETING}

Synthetic biology provides a powerful platform to assemble biological components into well-controlled systems to explore the intricate mechanisms underlying cellular behavior. Recently, a variety of microbial and plant-derived light-sensitive proteins have been engineered as optogenetic actuators to spatially and temporally control gene expression in various cells (Figure 6). Using different types of opsins and viral delivery vectors for devising new optogenetic tools, investigators can engineer cells to detect and respond to a variety of extracellular and intracellular signals to manipulate the cellular machinery towards novel purposes with precise spatiotemporal control [91-94]. For example, designable transcription factors such as zinc fingers (ZF) and transcription-activator like effectors (TALE) can be engineered to bind any DNA segment of choice. By combining the TALE technology with optogenetic control elements, very fast regulation of mammalian endogenous transcription could be achieved (Figure 6A) [95].

Another example, the light-inducible transcriptional effectors (LITEs) system consists of TALE DNA-binding domain from Xanthomonas sp. [96-98]. and the light- sensitive cryptochrome 2 (CRY2) protein from Arabidopsis thaliana (TALE-CRY2) [99] and its interactive partner CIB1-effector $[99,100]$. Upon blue light illumination, CRY2 changes its conformation and subsequently recruit CIB1-VP64 to the target promoter to regulate downstream gene expression. Furthermore, the LITE system has been applied to in vitro and in vivo tests. A Grm2-targeted LITE was applied into primary cortical neurons by adeno-associated viruses (AAVs) [95,101], which upregulated Grm2 mRNA and mGluR2 protein levels after light stimulation. Additionally, AAV vectors carrying TALE(Grm2)-CIB1 and CRY2PHR-VP64 were introduced into the prefrontal cortex (PFC), triggering light-dependent increase of Grm2 mRNA after illumination with light. The epigenetic mark-modified TALEhistone effector constructs (epiTALEs) have induced endogenous Grm 2 for interplay between epigenetic and transcriptional dynamics. This LITE system provides a powerful tool for engineering and modifying mammalian genomes.

\section{OPTOGENETIC NETWORKS FOR GENE EDITING}

A follow-up study by other groups demonstrated how to use light to control the powerful gene-editing tool-the clustered regularly interspaced short palindromic repeats (CRISPR) system [15,102-104]. In contrast to classical designer nucleases based on chimeric fusions between a ZF or TALE-domain to a non-specific endonuclease, the Cas9 protein is by nature a site-specific endonuclease. 
A

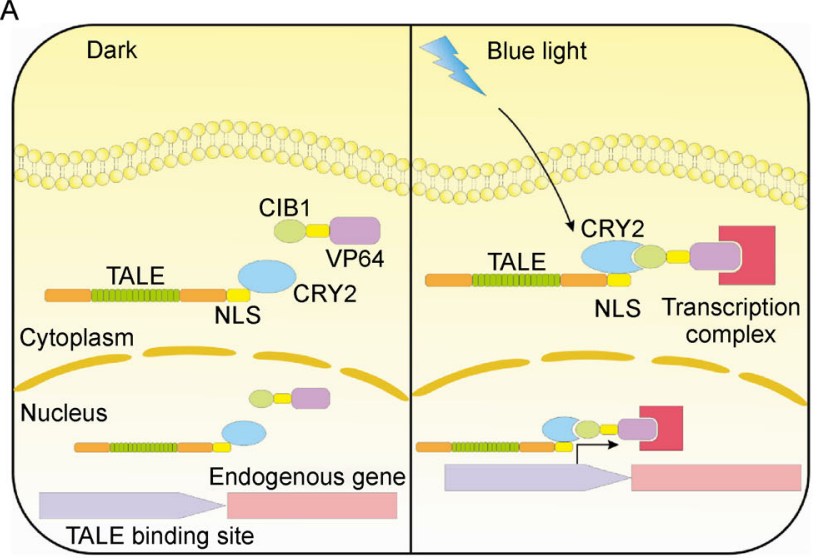

B

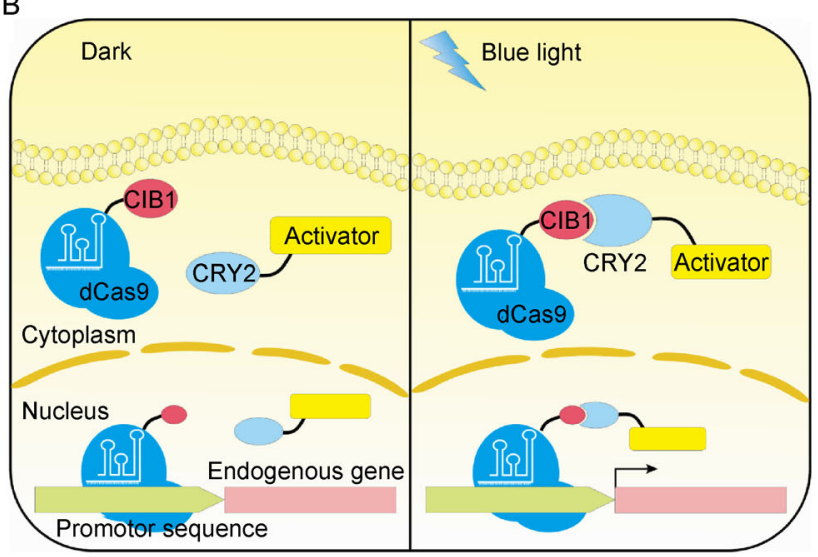

C

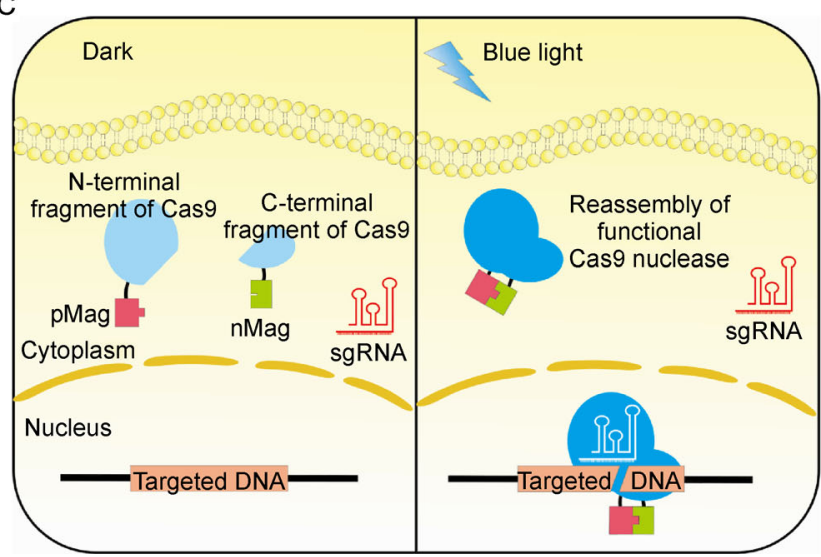

Figure 6. Optogenetic tools for Genome editing. (A) Optical control of mammalian endogenous transcription based on the LITE system. Light-inducible transcriptional effectors (LITEs) consisting of transcription activator-like effectors (TALEs), DNA-binding domain with the light-sensitive cryptochrome 2 (CRY2) protein and its interactive partner CIB1 from Arabidopsis thaliana. In the dark, TALE-CRY2 binds to the target gene promoter while the CIB1-effector remains inactivate. When illuminated with blue light, CRY2 conformation is changed and recruits CIB1-effector (VP64) to the target region to induce downstream gene expression. (B) Photoactivatable transcription system based on CRISPR-Cas9. This CRISPR-Cas9-based photoactivatable transcription system includes two fusion proteins and sgRNAs. One fusion protein contains dCas 9 and CIB1, and binds to the targeted genome sequence by sgRNAs, while the other fusion protein consists of the photolyase homology region of CRY2 (CRY2PHR) and the transcriptional activator domain. In the dark, the dCas9-CIB1 complex binds to the targeted gene promoter by sgRNAs while the activator probe is free in the nucleus and cannot activate the target genes. Upon blue light illumination, the downstream gene expression is activated by heterodimerized CRY2PHR and CIB1 and then the transcriptional activator domain is recruited to the target gene region. (C) Engineered photoactivatable Cas9 (paCas9) genome editing in human cells. Optically controlled Cas9 is based on the split Cas9 fragments fused with light-responsive dimerization domains (pMag and nMag). In the dark, Cas 9 is split into two fragments without gene editing. Under blue light illumination, pMag and nMag are heterodimerized to make the split Cas 9 fragments active, leading to resumption of RNA-guided nuclease activity to activate genome editing.

Using Cas9 and a set of user-defined guide RNA molecules (gRNA), specific DNA sequences and be targeted, cleaved and modified with unprecedented precision [105-107]. Now, methods for selectively deploying CRISPR have been developed with the flick of a light switch.

Hemphill et al. pioneered the development of genetically encoded light-activated Cas9 to investigate biological processes such as signal transduction and gene expression [103]. In this study, the light-sensitive lysine residue was inserted near a gRNA binding site on Cas9 proteins in human embryonic kidney cells. The modified lysine blocked gRNA binding, thereby preventing Cas9 from activating gene editing. Upon UV light exposure, however, the lysine changes shape to allow gRNA to bind to Cas9, thereby enabling gene editing. This lightactivated system can also be used to silence gene expression. 
Nihongaki et al. [102] have also developed a CRISPRCas9-based photoactivatable circuit based on an catalytically inactive Cas9 variant (dCas9), the light-sensitive cryptochrome 2 (CRY2) and its binding partner CIB1 from Arabidopsis thaliana [99], enabling blue lighttriggered activation of user-defined endogenous genes (Figure 6B). This optogenetic system can rapidly and reversibly activate the target gene, as well as various endogenous genes in mammalian cells, thus making optogenetic regulation of mammalian genome editing possible for various synthetic biology applications.

An engineered photoactivatable Cas9 (paCas9) system was also reported in human embryonic kidney cells by Nihongaki et al. [15]. In this study, paCas9 was generated from two split Cas9 fragments and light-responsive dimerization domains named Magnets, which could modify the targeted genome sequence through joining of both non-homologous ends and activation of homology-directed repair pathways under blue light irradiation (Figure 6C). Moreover, paCas9 can temporally and reversibly control gene editing in response to light. Several reports have shown that programming dCas9 to a transcription site can block RNA polymerase and transcription (termed CRISPR interference or CRISPRi) [108]. A photoactivatable CRISPRi (paCRISPRi) was designed to optically control RNA-guided transcription for targeted gene silencing.

\section{CONCLUSION AND PERSPECTIVES}

Synthetic biology provides a powerful platform to understand and harness biology, with influences from many scientific and engineering disciplines, and can exert a profound impact on various aspects of daily life and society in the future. Recently, two design principles are mainly applied for various light-inducible systems to control gene expression. One is based on light-dependent recruitment of transcriptional activation domain to DNAbound proteins, the other is based on light-modulated dimerization of a photoreceptor for reconstitution a DNAbinding domain to activate the target gene transcription. Nowadays, synthetic biologists have designed complex artificial gene circuits assembled into biosensing devices to monitor cellular behavior, which have promising therapeutic applications $[3,109,110]$. Compared to chemical or microwave systems, optogenetic tools triggered by specific wavelengths of light offer unprecedented spatiotemporal precision without the addition of chemical inducers that might perturb the system under investigation $[95,111]$. Consequently, the field of optogenetics has experienced a big upsurge over the last few years, and have begun to revolutionize biomedical research. Various optogenetic approaches have already been applied for numerous diseases, such as metabolic disorders, cancer, and immune diseases. A dual-input genetic circuit was reported to simultaneously modulated by utilizing the benefits of a chemical and light inducer. Moreover, the target gene of the reported circuits can be quantitatively expressed by regulating the chemical concentration and light irradiance both in the cells and mice [11].

Yet, can the optogenetic networks be validated outside the laboratory? To achieve the goals, standardized genetic circuits could be designed to ensure more robust and safe for patients. Moreover, most of the current genetic circuits were engineered into human embryonic kidney cells, the clinically proven mesenchymal stem cells from the patients could be used in clinic in the future. In addition, therapeutic proteins could be modulated upon light illumination in the patients.

To date, various optogenetic networks have been engineered to control biological processes using light. Two types of devices can perform these functions: lightdriven actuators control electrochemical signals, while light-emitting sensors report them. These range from light-inducible ion channels and receptors to the targeted interaction of proteins with light-induced proteolysis and light-induced second messenger products for recruitment of the related proteins. Most mammalian light-responsive gene circuits are controlled by blue light-responsive gene switches $[38,39,112]$. However, these are limited by lower efficiency in deep tissue. Red light systems could prove to be highly influential because of its selective targeting capacity and higher efficiency and viability $[29,113]$. However, some red light-responsive gene networks need phytochromobilin, which is not only difficult to acquire but also unlikely to be clinically licensed because of the side effects caused by this plantderived co-factor. Therefore, a more concerted effort towards advancing optogenetic devices in mammalian cells will be crucial for the next-generation of therapeutic switches, such as the choice of clinically safe light sources and/or using optogenetic systems that do not interfere with other metabolic processes. Multichromatic control circuits will further broaden the scope of light-controlled circuits used in biomedical applications.

Further development of optogenetic modules will be necessary to combine light-responsive gene expression systems with multi-spectral imaging without the need for exogenous compounds. Despite these challenges, future work using these novel tools and techniques will undoubtedly boost the development of next-generation synthetic biology in biotechnological applications. Lastly, optogenetic tools are maturing towards clinical medical practice, where they could induce therapeutic protein synthesis in patients subjected to light stimulation.

\section{ACKNOWLEDGEMENTS}

This work was financially supported by the National Key Research and 
Development Program of China, Stem Cell and Translational Research (No. 2016YFA0100300), by the National Natural Science Foundation of China (Nos. 31522017, 31470834 and 31670869), by the Science and Technology Commission of Shanghai Municipality (Nos.15QA1401500 and 14JC1401700), and by the Thousand Youth Talents Plan of China to H.Y.

\section{COMPLIANCE WITH ETHICS GUIDELINES}

The authors Meiyan Wang, Yuanhuan Yu, Jiawei Shao, Boon Chin Heng and Haifeng Ye declare they have no conflict of interest.

This article does not contain any studies with human or animal subjects performed by any of the authors.

\section{REFERENCES}

1. Church, G. M., Elowitz, M. B., Smolke, C. D., Voigt, C. A. and Weiss, R. (2014) Realizing the potential of synthetic biology. Nat. Rev. Mol. Cell Biol., 15, 289-294

2. Xie, M. Q., Haellman, V. and Fussenegger, M. (2016) Synthetic biology - application-oriented cell engineering. Curr. Opin. Biotechnol., 40, 139-148

3. Khalil, A. S. and Collins, J. J. (2010) Synthetic biology: applications come of age. Nat. Rev. Genet., 11, 367-379

4. Gardner, T. S., Cantor, C. R. and Collins, J. J. (2000) Construction of a genetic toggle switch in Escherichia coli. Nature, 403, 339342

5. Ye, H. F., Xie, M. Q., Xue, S., Hamri, G. C., Yin, J. L., Zulewski, H., and Fussenegger, M. (2016) Self-adjusting synthetic gene circuit for correcting insulin resistance. Nat. Biomed. Eng. 1, 0005

6. Stricker, J., Cookson, S., Bennett, M. R., Mather, W. H., Tsimring, L. S. and Hasty, J. (2008) A fast, robust and tunable synthetic gene oscillator. Nature, 456, 516-519

7. Fung, E., Wong, W. W., Suen, J. K., Bulter, T., Lee, S. G. and Liao, J. C. (2005) A synthetic gene-metabolic oscillator. Nature, $435,118-122$

8. Tabor, J. J., Salis, H. M., Simpson, Z. B., Chevalier, A. A., Levskaya, A., Marcotte, E. M., Voigt, C. A. and Ellington, A. D. (2009) A synthetic genetic edge detection program. Cell, 137, $1272-1281$

9. Bulter, T., Lee, S. G., Wong, W. W. C., Fung, E., Connor, M. R. and Liao, J. C. (2004) Design of artificial cell-cell communication using gene and metabolic networks. Proc. Natl. Acad. Sci. USA, 101, 2299-2304

10. Pathak, G. P., Vrana, J. D. and Tucker, C. L. (2013) Optogenetic control of cell function using engineered photoreceptors. Biol. Cell, 105, 59-72

11. Chen, X. J., Li, T., Wang, X., Du, Z. M., Liu, R. M. and Yang, Y. (2016) Synthetic dual-input mammalian genetic circuits enable tunable and stringent transcription control by chemical and light. Nucleic Acids Res., 44, 2677-2690

12. Chen, X., Li, T., Wang, X. and Yang, Y. (2015) A light-switchable bidirectional expression module allowing simultaneous regulation of multiple genes. Biochem. Biophys. Res. Commun., 465, 769-776

13. Kemmer, C., Gitzinger, M., Daoud-El Baba, M., Djonov, V.,
Stelling, J. and Fussenegger, M. (2010) Self-sufficient control of urate homeostasis in mice by a synthetic circuit. Nat. Biotechnol., $28,355-360$

14. Rössger, K., Charpin-El Hamri, G. and Fussenegger, M. (2013) Reward-based hypertension control by a synthetic brain-dopamine interface. Proc. Natl. Acad. Sci. USA, 110, 18150-18155

15. Nihongaki, Y., Kawano, F., Nakajima, T. and Sato, M. (2015) Photoactivatable CRISPR-Cas9 for optogenetic genome editing. Nat. Biotechnol., 33, 755-760

16. Zhang, K. and Cui, B. X. (2015) Optogenetic control of intracellular signaling pathways. Trends Biotechnol., 33, 92-100

17. Weitzman, M. and Hahn, K. M. (2014) Optogenetic approaches to cell migration and beyond. Curr. Opin. Cell Biol., 30, 112-120

18. Okuno, D., Asaumi, M. and Muneyuki, E. (1999) Chloride concentration dependency of the electrogenic activity of halorhodopsin. Biochemistry, 38, 5422-5429

19. Zemelman, B. V., Lee, G. A., Ng, M. and Miesenbock, G. (2002) Selective photostimulation of genetically ChARGed neurons. Neuron, 33, 15-22

20. Nagel, G., Szellas, T., Huhn, W., Kateriya, S., Adeishvili, N., Berthold, P., Ollig, D., Hegemann, P. and Bamberg, E. (2003) Channelrhodopsin-2, a directly light-gated cation-selective membrane channel. Proc. Natl. Acad. Sci. USA, 100, 13940-13945

21. Boyden, E. S., Zhang, F., Bamberg, E., Nagel, G. and Deisseroth, K. (2005) Millisecond-timescale, genetically targeted optical control of neural activity. Nat. Neurosci., 8, 1263-1268

22. Kato, H. E., Zhang, F., Yizhar, O., Ramakrishnan, C., Nishizawa, T., Hirata, K., Ito, J., Aita, Y., Tsukazaki, T., Hayashi, S., et al. (2012) Crystal structure of the channelrhodopsin light-gated cation channel. Nature, 482, 369-374

23. Oesterhelt, D. (1998) The structure and mechanism of the family of retinal proteins from halophilic archaea. Curr. Opin. Struct. Biol., 8, 489-500

24. Haupts, U., Tittor, J. and Oesterhelt, D. (1999) Closing in on bacteriorhodopsin: progress in understanding the molecule. Annu. Rev. Biophys. Biomol. Struct., 28, 367-399

25. Nagel, G., Brauner, M., Liewald, J. F., Adeishvili, N., Bamberg, E. and Gottschalk, A. (2005) Light activation of channelrhodopsin-2 in excitable cells of Caenorhabditis elegans triggers rapid Behavioral responses. Curr. Biol., 15, 2279-2284

26. Editorial (2011) Method of the Year 2010. Nat. Methods, 8, 1

27. News, S. (2010) Stepping away from the trees for a look at the forest. Science, 330, 1612-1613

28. Christie, J. M., Gawthorne, J., Young, G., Fraser, N. J. and Roe, A. J. (2012) LOV to BLUF: flavoprotein contributions to the optogenetic toolkit. Mol. Plant, 5, 533-544

29. Muller, K., Engesser, R., Metzger, S., Schulz, S., Kampf, M. M., Busacker, M., Steinberg, T., Tomakidi, P., Ehrbar, M., Nagy, F., et al. (2013) A red/far-red light-responsive bi-stable toggle switch to control gene expression in mammalian cells. Nucleic Acids Res., 41, e77

30. Wieland, M., Muller, M., Kyburz, A., Heissig, P., Wekenmann, S., Stolz, F., Auslander, S. and Fussenegger, M. (2014) Engineered UV-A light-responsive gene expression system for 
measuring sun cream efficacy in mammalian cell culture. J. Biotechnol., 189, 150-153

31. Kawano, F., Suzuki, H., Furuya, A. and Sato, M. (2015) Engineered pairs of distinct photoswitches for optogenetic control of cellular proteins. Nat. Commun., 6,6256

32. Folcher, M., Oesterle, S., Zwicky, K., Thekkottil, T., Heymoz, J., Hohmann, M., Christen, M., Daoud-El-Baba, M. D., Buchmann, P. and Fussenegger, M. (2014) Mind-controlled transgene expression by a wireless-powered optogenetic designer cell implant. Nat. Commun., 5, 5392

33. Fraikin, G. Y., Strakhovskaya, M. G., Belenikina, N. S. and Rubin, A. B. (2015) Bacterial photosensory proteins: regulatory functions and optogenetic applications. Microbiology, 84, 461472

34. Montgomery, K. L., Iyer, S. M., Christensen, A. J., Deisseroth, K. and Delp, S. L. (2016) Beyond the brain: optogenetic control in the spinal cord and peripheral nervous system. Sci. Transl. Med., 8, 337rv5

35. Laakso, M. and Kuusisto, J. (2014) Insulin resistance and hyperglycaemia in cardiovascular disease development. Nat. Rev. Endocrinol., 10, 293-302

36. Chen, L., Magliano, D. J. and Zimmet, P. Z. (2012) The worldwide epidemiology of type 2 diabetes mellitus - present and future perspectives. Nat. Rev. Endocrinol., 8, 228-236

37. Grundy, S. M. (2006) Drug therapy of the metabolic syndrome: minimizing the emerging crisis in polypharmacy. Nat. Rev. Drug Discov., 5, 295-309

38. Ye, H. F., Doaud-El Baba, M., Peng, R. W. and Fussenegger, M. (2011) A synthetic optogenetic transcription device enhances blood-glucose homeostasis in mice. Science, 332, 1565-1568

39. Wang, X., Chen, X. J. and Yang, Y. (2012) Spatiotemporal control of gene expression by a light-switchable transgene system. Nat. Methods, 9, 266-269

40. Waldmann, T. A. (2003) Immunotherapy: past, present and future. Nat. Med., 9, 269-277

41. Kalos, M. and June, C. H. (2013) Adoptive T cell transfer for cancer immunotherapy in the era of synthetic biology. immunity, $39,49-60$

42. Restifo, N. P., Dudley, M. E. and Rosenberg, S. A. (2012) Adoptive immunotherapy for cancer: harnessing the $\mathrm{T}$ cell response. Nat. Rev. Immunol., 12, 269-281

43. Vivier, E., Ugolini, S., Blaise, D., Chabannon, C. and Brossay, L. (2012) Targeting natural killer cells and natural killer T cells in cancer. Nat. Rev. Immunol., 12, 239-252

44. He, L., Zhang, Y. W., Ma, G. L., Tan, P., Li, Z. J., Zang, S. B., Wu, X., Jing, J., Fang, S. H., Zhou, L. J., et al. (2015) Near-infrared photoactivatable control of $\mathrm{Ca}^{2+}$ signaling and optogenetic immunomodulation. eLife, 4, 25

45. Xu, Y. X., Hyun, Y. M., Lim, K., Lee, H., Cummings, R. J., Gerber, S. A., Bae, S., Cho, T. Y., Lord, E. M. and Kim, M. (2014) Optogenetic control of chemokine receptor signal and T-cell migration. Proc. Natl. Acad. Sci. USA, 111, 6371-6376

46. Boissonnas, A., Fetler, L., Zeelenberg, I. S., Hugues, S. and Amigorena, S. (2007) In vivo imaging of cytotoxic $\mathrm{T}$ cell infiltration and elimination of a solid tumor. J. Exp. Med., 204, 345-356

47. Breart, B., Lemaitre, F., Celli, S. and Bousso, P. (2008) Twophoton imaging of intratumoral $\mathrm{CD} 8^{+} \mathrm{T}$ cell cytotoxic activity during adoptive $\mathrm{T}$ cell therapy in mice. J. Clin. Invest., 118, 1390-1397

48. Cipolotti, L., Shallice, T., Chan, D., Fox, N., Scahill, R., Harrison, G., Stevens, J. and Rudge, P. (2001) Long-term retrograde amnesia ... the crucial role of the hippocampus. Neuropsychologia, 39, 151-172

49. Nadel, L. and Moscovitch, M. (1997) Memory consolidation, retrograde amnesia and the hippocampal complex. Curr. Opin. Neurobiol., 7, 217-227

50. Buccione, I., Fadda, L., Serra, L., Caltagirone, C. and Carlesimo, G. A. (2008) Retrograde episodic and semantic memory impairment correlates with side of temporal lobe damage. J. Int. Neuropsychol. Soc., 14, 1083-1094

51. Liu, X., Ramirez, S., Pang, P. T., Puryear, C. B., Govindarajan, A., Deisseroth, K. and Tonegawa, S. (2012) Optogenetic stimulation of a hippocampal engram activates fear memory recall. Nature, 484, 381-385.

52. Ramirez, S., Liu, X., Lin, P. A., Suh, J., Pignatelli, M., Redondo, R. L., Ryan, T. J. and Tonegawa, S. (2013) Creating a false memory in the hippocampus. Science, 341, 387-391

53. Redondo, R. L., Kim, J., Arons, A. L., Ramirez, S., Liu, X. and Tonegawa, S. (2014) Bidirectional switch of the valence associated with a hippocampal contextual memory engram. Nature, 513, 426-430

54. Denny, C. A., Kheirbek, M. A., Alba, E. L., Tanaka, K. F., Brachman, R. A., Laughman, K. B., Tomm, N. K., Turi, G. F., Losonczy, A. and Hen, R. (2014) Hippocampal memory traces are differentially modulated by experience, time, and adult neurogenesis. Neuron, 83, 189-201

55. Tanaka, K. Z., Pevzner, A., Hamidi, A. B., Nakazawa, Y., Graham, J. and Wiltgen, B. J. (2014) Cortical representations are reinstated by the hippocampus during memory retrieval. Neuron, $84,347-354$

56. Cowansage, K. K., Shuman, T., Dillingham, B. C., Chang, A., Golshani, P. and Mayford, M. (2014) Direct reactivation of a coherent neocortical memory of context. Neuron, 84, 432-441

57. Ryan, T. J., Roy, D. S., Pignatelli, M., Arons, A. and Tonegawa, S. (2015) Engram cells retain memory under retrograde amnesia. Science, 348, 1007-1013

58. Nader, K., Schafe, G. E. and Le Doux, J. E. (2000) Fear memories require protein synthesis in the amygdala for reconsolidation after retrieval. Nature, 406, 722-726

59. Schroll, C., Riemensperger, T., Bucher, D., Ehmer, J., Voller, T., Erbguth, K., Gerber, B., Hendel, T., Nagel, G., Buchner, E., et al. (2006) Light-induced activation of distinct modulatory neurons triggers appetitive or aversive learning in Drosophila larvae. Curr. Biol., 16, 1741-1747

60. Bellmann, D., Richardt, A., Freyberger, R., Nuwal, N., Schwarzel, M., Fiala, A. and Stortkuhl, K. F. (2010) Optogenetically induced olfactory stimulation in Drosophila larvae 
reveals the neuronal basis of odor-aversion behavior. Front. Behav. Neurosci., 4, 10

61. Hull, E. M., Du, J. F., Lorrain, D. S. and Matuszewich, L. (1995) Extracellular dopamine in the medial preoptic area - implications for sexual motivation and hormonal-control of copulation. J. Neurosci., 15, 7465-7471

62. Sadeghipour, H., Ghasemi, M., Ebrahimi, F. and Dehpour, A. R. (2007) Effect of lithium on endothelium-dependent and neurogenic relaxation of rat corpus cavernosum: role of nitric oxide pathway. Nitric Oxide, 16, 54-63

63. Melis, M. R., Spano, M. S., Succu, S. and Argiolas, A. (2000) Activation of $\gamma$-aminobutyric acid $_{\mathrm{A}}$ receptors in the paraventricular nucleus of the hypothalamus reduces apomorphine-, $\mathrm{N}$ methyl-D-aspartic acid- and oxytocin-induced penile erection and yawning in male rats. Neurosci. Lett., 281, 127-130

64. Melis, M. R., Succu, S. and Argiolas, A. (1997) Prevention by morphine of $N$-methyl-D-aspartic acid-induced penile erection and yawning: involvement of nitric oxide. Brain Res. Bull., 44, 689-694

65. Melis, M. R., Succu, S., Iannucci, U. and Argiolas, A. (1997) Nmethyl-D-aspartic acid-induced penile erection and yawning: role of hypothalamic paraventricular nitric oxide. Eur. J. Pharmacol., 328, 115-123

66. Toda, N., Ayajiki, K. and Okamura, T. (2005) Nitric oxide and penile erectile function. Pharmacol. Ther., 106, 233-266

67. Melis, M. R. and Argiolas, A. (1999) Yawning: role of hypothalamic paraventricular nitric oxide. Acta Pharmacol. Sin., 20, 778-788

68. Melis, M. R. and Argiolas, A. (1997) Role of central nitric oxide in the control of penile erection and yawning. Prog. Neuropsychopharmacol. Biol. Psychiatry, 21, 899-922

69. Nunes, K. P., Labazi, H. and Webb, R. C. (2012) New insights into hypertension-associated erectile dysfunction. Curr. Opin. Nephrol. Hypertens., 21, 163-170

70. Fraga-Silva, R. A., Costa-Fraga, F. P., Faye, Y., Sturny, M., Santos, R. A., Dasilva, R. F. and Stergiopulos, N. (2014) An increased arginase activity is associated with corpus cavernosum impairment induced by hypercholesterolemia. J. Sex. Med., 11, 1173-1181

71. Pauker-Sharon, Y., Arbel, Y., Finkelstein, A., Halkin, A., Herz, I., Banai, S. and Justo, D. (2013) Cardiovascular risk factors in men with ischemic heart disease and erectile dysfunction. Urology, 82, 377-381

72. Huang, S. S., Lin, C. H., Chan, C. H., Loh, E. W. and Lan, T. H. (2013) Newly diagnosed major depressive disorder and the risk of erectile dysfunction: a population-based cohort study in Taiwan. Psychiatry Res., 210, 601-606

73. Kim, T., Folcher, M., Doaud-El Baba, M. and Fussenegger, M. (2015) A synthetic erectile optogenetic stimulator enabling bluelight-inducible penile erection. Angew. Chem. Int. Ed., 54, 59335938

74. Champion, H. C., Bivalacqua, T. J., Hyman, A. L., Ignarro, L. J., Hellstrom, W. J. G. and Kadowitz, P. J. (1999) Gene transfer of endothelial nitric oxide synthase to the penis augments erectile responses in the aged rat. Proc. Natl. Acad. Sci. USA, 96, 1164811652

75. Bennett, N. E., Kim, J. H., Wolfe, D. P., Sasaki, K., Yoshimura, N., Goins, W. F., Huang, S., Nelson, J. B., de Groat, W. C. and Glorioso, J. C. (2005) Improvement in erectile dysfunction after neurotrophic factor gene therapy in diabetic rats. J. Urol., 173 1820-1824

76. Christ, G. J., Day, N., Santizo, C., Sato, Y. S., Zhao, W. X., Sclafani, T., Bakal, R., Salman, M., Davies, K. and Melman, A. (2004) Intracorporal injection of hSlo cDNA restores erectile capacity in STZ-diabetic F-344 rats in vivo. Heart Circul. Physiol., 287, H1544-H1553

77. Magee, T. R., Ferrini, M., Garban, H. J., Vernet, D., Mitani, K., Rajfer, J. and Gonzalez-Cadavid, F. (2002) Gene therapy of erectile dysfunction in the rat with penile neuronal nitric oxide synthase. Biol. Reprod., 67, 20-28

78. Chancellor, M. B., Tirney, S., Mattes, C. E., Tzeng, E., Birder, L. A., Kanai, A. J., de Groat, W. C., Huard, J. and Yoshimura, N. (2003) Nitric oxide synthase gene transfer for erectile dysfunction in a rat model. BJU Int., 91, 691-696

79. Antonenkov, V. D., Grunau, S., Ohlmeier, S. and Hiltunen, J. K. (2010) Peroxisomes are oxidative organelles. Antioxid. Redox Signal., 13, 525-537

80. Millecamps, S. and Julien, J. P. (2013) Axonal transport deficits and neurodegenerative diseases. Nat. Rev. Neurosci., 14, 161176

81. Niopek, D., Benzinger, D., Roensch, J., Draebing, T., Wehler, P., Eils, R. and Di Ventura, B. (2014) Engineering light-inducible nuclear localization signals for precise spatiotemporal control of protein dynamics in living cells. Nat. Commun., 5, 4404

82. Crefcoeur, R. P., Yin, R. H., Ulm, R. and Halazonetis, T. D. (2013) Ultraviolet-B-mediated induction of protein-protein interactions in mammalian cells. Nat. Commun., 4,1779

83. Yang, X. J., Jost, A. P. T., Weiner, O. D. and Tang, C. (2013) A light-inducible organelle-targeting system for dynamically activating and inactivating signaling in budding yeast. Mol. Biol. Cell, 24, 2419-2430

84. Yumerefendi, H., Dickinson, D. J., Wang, H., Zimmerman, S. P., Bear, J. E., Goldstein, B., Hahn, K. and Kuhlman, B. (2015) Control of protein activity and cell fate specification via lightmediated nuclear translocation. PLoS One, 10, e0128443

85. Spiltoir, J. I., Strickland, D., Glotzer, M. and Tucker, C. L. (2016) Optical control of peroxisomal trafficking. ACS Synth. Biol., 5, $554-560$

86. van Bergeijk, P., Adrian, M., Hoogenraad, C. C. and Kapitein, L. C. (2015) Optogenetic control of organelle transport and positioning. Nature, 518, 111-114

87. Ishikawa, K., Tamura, Y. and Maruta, S. (2014) Photocontrol of mitotic kinesin Eg5 facilitated by thiol-reactive photochromic molecules incorporated into the loop L5 functional loop. J. Biochem., 155, 195-206

88. Kamei, T., Fukaminato, T. and Tamaoki, N. (2012) A photochromic ATP analogue driving a motor protein with reversible light-controlled motility: controlling velocity and binding manner 
of a kinesin-microtubule system in an in vitro motility assay. Chem. Commun. (Camb.), 48, 7625-7627

89. Nakamura, M., Chen, L., Howes, S. C., Schindler, T. D., Nogales, E. and Bryant, Z. (2014) Remote control of myosin and kinesin motors using light-activated gearshifting. Nat. Nanotechnol., 9, 693-697

90. Duan, L. T., Che, D., Zhang, K., Ong, Q. X., Guo, S. L. and Cui, B. X. (2015) Optogenetic control of molecular motors and organelle distributions in cells. Chem. Biol., 22, 671-682

91. Jena, B., Dotti, G. and Cooper, L. J. N. (2010) Redirecting T-cell specificity by introducing a tumor-specific chimeric antigen receptor. Blood, 116, 1035-1044

92. Yazawa, M., Sadaghiani, A. M., Hsueh, B. and Dolmetsch, R. E. (2009) Induction of protein-protein interactions in live cells using light. Nat. Biotechnol., 27, 941-945

93. Park, J. S., Rhau, B., Hermann, A., McNally, K. A., Zhou, C., Gong, D., Weiner, O. D., Conklin, B. R., Onuffer, J. and Lim, W. A. (2014) Synthetic control of mammalian-cell motility by engineering chemotaxis to an orthogonal bioinert chemical signal. Proc. Natl. Acad. Sci. USA, 111, 5896-5901

94. Taslimi, A., Zoltowski, B., Miranda, J. G., Pathak, G. P., Hughes, R. M. and Tucker, C. L. (2016) Optimized second-generation CRY2-CIB dimerizers and photoactivatable cre recombinase. Nat. Chem. Biol., 12, 425-430

95. Konermann, S., Brigham, M. D., Trevino, A. E., Hsu, P. D., Heidenreich, M., Cong, L., Platt, R. J., Scott, D. A., Church, G. M. and Zhang, F. (2013) Optical control of mammalian endogenous transcription and epigenetic states. Nature, 500, 472.

96. Boch, J., Scholze, H., Schornack, S., Landgraf, A., Hahn, S., Kay, S., Lahaye, T., Nickstadt, A. and Bonas, U. (2009) Breaking the code of DNA binding specificity of TAL-type III effectors. Science, 326, 1509-1512

97. Moscou, M. J. and Bogdanove, A. J. (2009) A simple cipher governs DNA recognition by TAL effectors. Science, 326, 1501

98. Zhang, F., Cong, L., Lodato, S., Kosuri, S., Church, G. M. and Arlotta, P. (2011) Efficient construction of sequence-specific TAL effectors for modulating mammalian transcription. Nat. Biotechnol., 29, 149-153

99. Kennedy, M. J., Hughes, R. M., Peteya, L. A., Schwartz, J. W., Ehlers, M. D. and Tucker, C. L. (2010) Rapid blue-light-mediated induction of protein interactions in living cells. Nat. Methods, 7, 973-975

100. Liu, H. T., Yu, X. H., Li, K. W., Klejnot, J., Yang, H. Y., Lisiero, D. and Lin, C. T. (2008) Photoexcited CRY2 interacts with CIB1 to regulate transcription and floral initiation in Arabidopsis. Science, 322, 1535-1539
101. Holkers, M., Maggio, I., Liu, J., Janssen, J. M., Miselli, F., Mussolino, C., Recchia, A., Cathomen, T. and Goncalves, M. (2013) Differential integrity of TALE nuclease genes following adenoviral and lentiviral vector gene transfer into human cells. Nucleic Acids Res., 41, e63

102. Nihongaki, Y., Yamamoto, S., Kawano, F., Suzuki, H. and Sato, M. (2015) CRISPR-Cas9-based photoactivatable transcription system. Chem. Biol., 22, 169-174

103. Hemphill, J., Borchardt, E. K., Brown, K., Asokan, A. and Deiters, A. (2015) Optical control of CRISPR/Cas9 gene editing. J. Am. Chem. Soc., 137, 5642-5645

104. Lin, F., Dong, L., Wang, W., Liu, Y., Huang, W. and Cai, Z. (2016) An efficient light-inducible P53 expression system for inhibiting proliferation of bladder cancer cell. Int. J. Biol. Sci., 12, 1273-1278

105. Schiml, S., Fauser, F. and Puchta, H. (2014) The CRISPR/Cas system can be used as nuclease for in planta gene targeting and as paired nickases for directed mutagenesis in Arabidopsis resulting in heritable progeny. Plant J., 80, 1139-1150

106. Ran, F. A., Hsu, P. D., Lin, C. Y., Gootenberg, J. S., Konermann, S., Trevino, A. E., Scott, D. A., Inoue, A., Matoba, S., Zhang, Y., et al. (2013) Double nicking by RNA-guided CRISPR Cas9 for enhanced genome editing specificity. Cell, 154, 1380-1389

107. Slaymaker, I. M., Gao, L. Y., Zetsche, B., Scott, D. A., Yan, W. X. and Zhang, F. (2016) Rationally engineered Cas9 nucleases with improved specificity. Science, 351, 84-88

108. Qi, L. S., Larson, M. H., Gilbert, L. A., Doudna, J. A., Weissman, J. S., Arkin, A. P. and Lim, W. A. (2013) Repurposing CRISPR as an RNA-guided platform for sequence-specific control of gene expression. Cell, 152, 1173-1183

109. Weber, W. and Fussenegger, M. (2012) Emerging biomedical applications of synthetic biology. Nat. Rev. Genet., 13, 21-35.

110. Xue, S., Yin, J. L., Shao, J. W., Yu, Y. H., Yang, L. F., Wang, Y. D., Xie, M. Q., Fussenegger, M. and Ye, H. F. (2016) A synthetic biology-inspired therapeutic strategy for targeting and treating hepatogenous diabetes. Mol. Ther., 443-455

111. Muller, K., Engesser, R., Metzger, S., Schulz, S., Kampf, M. M., Busacker, M., Steinberg, T., Tomakidi, P., Ehrbar, M., Nagy, F., et al. (2013) A red/far-red light-responsive bi-stable toggle switch to control gene expression in mammalian cells. Nucleic Acids Res., 41, e77

112. Motta-Mena, L. B., Reade, A., Mallory, M. J., Glantz, S., Weiner, O. D., Lynch, K. W. and Gardner, K. H. (2014) An optogenetic gene expression system with rapid activation and deactivation kinetics. Nat. Chem. Biol., 10, 196-202

113. Müller, K., Zurbriggen, M. D. and Weber, W. (2014) Control of gene expression using a red- and far-red light-responsive bi-stable toggle switch. Nat. Protoc., 9, 622-632 